\title{
Erratum to: Paraplegia after posterior only correction of congenital kyphosis
}

\author{
Claudio Lamartina $\cdot$ Pedro Berjano
}

Published online: 19 October 2011

(C) Springer-Verlag 2011

Erratum to: Eur Spine J (2011) 20:1582-1583,

\section{DOI 10.1007/s00586-011-1942-z}

The following affiliation was omitted:

I.R.C.C.S Istituto Ortopedico Galeazzi, Milan, Italy.

e-mail: c.lamartina@chirurgiavertebrale.net

The online version of the original article can be found under doi:10.1007/s00586-011-1942-z.

C. Lamartina $(\square) \cdot$ P. Berjano I.R.C.C.S Istituto Ortopedico Galeazzi, Milan, Italy

e-mail: c.lamartina@chirurgiavertebrale.net 